BULL. AUSTRAL. MATH. SOC.

VOL. $21(1980), 93-105$.

\title{
EXTENSION WITH LARGER NORM AND SEPARATION WITH DOUBLE SUPPORT \\ IN NORMED LINEAR SPACES
}

\author{
IVAN SINGER
}

\begin{abstract}
We prove, in normed linear spaces, the existence of extensions of continuous linear functionals from linear subspaces to the whole space, with arbitrarily prescribed larger norm. Also, we prove that under an additional boundedness assumption, in the known separation theorems for convex sets, there exist hyperplanes which separate and support both sets.
\end{abstract}

\section{Introduction}

If $G$ is a linear subspace of a normed linear space $E$ and $\varphi$ a continuous linear functional on $G$, the classical Hahn-Banach theorem states that there exists an extension of $\varphi$ to a continuous linear functional $\Phi$ on the whole space $E$ (that is, $\left.\Phi\right|_{G}=\varphi$ ), with the same norm $\|\Phi\|=\|\varphi\|$. Since obviously every extension $\Phi$ of $\varphi$, to the whole space $E$, has norm $\|\Phi\| \geq\|\varphi\|$, this theorem states, in other words, the existence of an extension $\Phi$ of $\varphi$, to the whole space $E$, with the smallest possible norm; however, no information is given about the existence of an extension $\Phi$ with a prescribed norm $\lambda$, where $\lambda>\|\varphi\|$; that is, of an extension with a prescribed larger norm. Furthermore, the known separation theorems for convex sets do not give information about "how non-strict" separation can be, more precisely, about the existence of functionals or hyperplanes which not only separate, but at the same time also support, both sets (such a property may be called "separation with

Received 2 August 1979. 
double support"); for a survey of known results on separation and support properties of convex sets, see [4].

In the present paper we shall show that the answer to the above extension problem and, under a certain additional boundedness assumption, the answer to the above problem on separation with double support, are affirmative. Let us point out that we have been led to these results by the study of an optimization problem, namely, that of the minimization of continuous linear functionals on caverns and that we have applied our Corollary 2 to solve this problem ([8], Theorems 2.2 and 3.3).

In order to be able to apply our results on extension with larger norm to separation with double support, we shall give these extension theorems for an arbitrary asymmetric norm. We recall (see, for example, [6], [2]) that a non-negative functional $x \rightarrow\|x\|$ on a linear space $E$ is called an asymmetric norm on $E$, if

(a) $\|x\|=0$ if and oniy if $x=0$;

(b) $\|x+y\| \leq\|x\|+\|y\| \quad(x, y \in E)$;

(c) $\|\alpha x\|=\alpha\|x\| \quad(x \in E, \alpha \geq 0)$;

property (c) is called the positive homogeneity of $\|\cdot\|$. Thus, in particular, every norm in the usual sense, that is, every "symmetric" norm (that is, such that $\|\alpha x\|=|\alpha|\|x\|$ for all $x \in E$ and $\alpha \in R$, the real line) is an asymmetric norm. We shall say that an asymmetric norm $\|\cdot\|$ on a normed linear space $E=(E,|\cdot|)$ is equivalent to the initial norm on $E$ (or, briefly, $\|\cdot\|$ is an equivalent asymmetric norm on $E$ ), if there exist two constants $C_{1}, C_{2}>0$ such that $C_{1}|x| \leq\|x\| \leq C_{2}|x|$ $(x \in E)$; then, clearly, $|\cdot|$ and $\|\cdot\|$ generate the same topology on $E$, via the (usual) distance dist $|\cdot|(x, y)=|x-y|$ and the asymmetric distance dist $\|\cdot\|(x, y)=\|x-y\|$ respectively. For a linear space $E$, an asymmetric norm $\|\cdot\|$ on $E$, and any linear functional $\Phi$ on $E$, we shall consider the asymetric norm $\|\Phi\|=\sup _{x \in E} \Phi(x)$ and we shall denote $x \in E$ $\|x\| \leq 1$

by $E^{*}$ the set of all linear functionals $\Phi$ on $E$ such that $\|\Phi\|<+\infty$. If $G$ is a linear subspace of $E$, we shall denote $G^{\underline{1}}=\left\{\Phi \in E^{*}|\Phi|_{G}=0\right\}$. 
We also recall some other notations and notions which we shall use in the sequel. For a subset $A$ of a normed linear space $(E,|\cdot|)$, we shall denote by $\bar{A}$ and Int $A$ the closure and the interior of $A$ respectively (in the norm topology of $E$ ). A linear manifold $V$ in $E$ is a subset of the form $V=x_{0}+G=\left\{x_{0}+g \mid g \in G\right\}$, where $G$ is a linear subspace of $E$ and $x_{0} \in E$. A hyperplane $H$ (by "hyperplane" we shall always mean a closed hyperplane) is said to support a set $A$ in $E$, if $A$ lies in one of the two closed half-spaces determined by $H$ and

$$
\operatorname{dist}|\cdot|^{(H, A)=} \inf _{\substack{y \in H \\ z \in A}}|y-z|=0
$$

(thus, we do not require that $H \cap A \neq \emptyset$ ). For $H=\{y \in E \mid \Phi(y)=c\}$, where $\Phi \in E^{*}, \Phi \neq 0$ and $c \in R$, this happens if and only if either $c=\sup \Phi(A)$ or $c=$ inf $\Phi(A)$, so every hyperplane $H$ supporting $A$ can by written (replacing, if necessary, $\Phi$ by $-\Phi$ ) in the form $H=\{y \in E \mid \Phi(y)=\sup \Phi(A)\}$, where $\Phi \in E^{*}, \Phi \neq 0$ (and, conversely, every hyperplane of this form, with sup $\Phi(A)<+\infty$, supports $A$ ).

Finally, we note that, while many of the known theorems on separation of convex sets are given in topological linear spaces, in the case of our results on separation with double support this would yield no gain in generality, since by virtue of Kolmogorov's theorem on normability (see, for example, [1], Chapter I, \$4, (12) (c)), every topological linear space $E$ in which there exists a bounded convex subset with non-empty interior, must be normable. Therefore, we shall give our results on separation with double support only in normed linear spaces $(E,|\cdot|$ ) (although, in the first parts of these results, the norm $|\cdot|$ will not occur explicitly).

\section{Extension with larger norm}

THEOREM 1. Let $E$ be a linear space, $\|\cdot\|$ con asymmetric norm on $E, G$ a linear subspace of $E$ with $G^{\underline{1}} \neq\{0\}, \varphi \in G^{*}$ and $\lambda \geq\|\varphi\|$. Then there exists $\Phi \in E^{*}$ such that

$$
\left.\Phi\right|_{G}=\varphi,\|\Phi\|=\lambda \text {. }
$$

Moreover, if $\lambda>\|\varphi\|$, then there exist at least two distinct functionals $\Phi \in E^{*}$ satisfying (1). 
Proof. By the Hahn-Banach theorem for asymmetric norms (see, for example, [5], Chapter III, §17), there exists $\Phi_{1} \in E^{*}$ such that

$$
\left.\Phi_{1}\right|_{G}=\varphi, \quad\left\|\Phi_{1}\right\|=\|\varphi\| \text {. }
$$

Furthermore, since $G^{\frac{1}{}} \neq\{0\}$, there exists $\Phi_{2} \in E^{*}$ such that

$$
\left.\Phi_{2}\right|_{G}=\varphi, \Phi_{2} \neq \Phi_{1}
$$

indeed, one can take $\Phi_{2}=\Phi_{1}+\Phi_{0}$, where $\Phi_{0} \in G^{\perp}, \Phi_{0} \neq 0$. Let

$$
\begin{aligned}
\Psi_{\alpha} & =\alpha \Phi_{1}+(1-\alpha) \Phi_{2}=\alpha\left(\Phi_{1}-\Phi_{2}\right)+\Phi_{2} \quad(\alpha \in R), \\
\chi(\alpha) & =\left\|\Psi_{\alpha}\right\| \quad(\alpha \in R) .
\end{aligned}
$$

Then $X$ is continuous on $R, X(\alpha) \rightarrow+\infty$ as $\alpha \rightarrow \pm \infty$, and, by (2), $X(1)=\left\|\Psi_{1}\right\|=\left\|\Phi_{1}\right\|=\|\varphi\| \leq \lambda<+\infty$. Consequently, there exists $\alpha_{1} \in R$ such that $\chi\left(\alpha_{1}\right)=\lambda$ and, if $\lambda>\|\varphi\|$, then there exist $\alpha_{1}>1$ and $\alpha_{2}<1$ such that $x\left(\alpha_{1}\right)=x\left(\alpha_{2}\right)=\lambda$. Then, clearly, the functional $\Phi=\Psi_{\alpha_{1}}$ (respectively, if $\lambda>\|\varphi\|$, then both $\Phi=\Psi_{\alpha_{1}}$ and $\Phi=\Psi_{\alpha_{2}}$ ) satisfies (1), which completes the proof of Theorem 1 .

Even when there are an infinity of distinct functionals $\Phi \in E^{*}$ such that $\left.\Phi\right|_{G}=\varphi,\|\Phi\|=\|\varphi\|$, it may happen that for each $\lambda>\|\varphi\|$ there exist exactly two distinct functionals $\Phi \in E^{*}$ satisfying (I), as shown by

EXAMPLE 1. Let $E=i_{2}^{\infty}$, the two-dimensional space with the norm $\left\|\left(\xi_{1}, \xi_{2}\right)\right\|=\max \left(\left|\xi_{1}\right|,\left|\xi_{2}\right|\right)$, let $G=\left\{\left(\xi_{1}, \xi_{2}\right) \in E \mid \xi_{1}=\xi_{2}\right\}$ and define $\varphi \in G^{*}$ by $\varphi\left(\left(\xi_{1}, \xi_{2}\right)\right)=\xi_{1}\left(\left(\xi_{1}, \xi_{2}\right) \in G\right)$. Then $\|\varphi\|=1$ and each $\Phi \in E^{*}$ satisfying $\left.\Phi\right|_{G}=\varphi$ is of the form

$$
\Phi_{\alpha}\left(\left(\xi_{1}, \xi_{2}\right)\right)=\alpha \xi_{1}+(1-\alpha) \xi_{2}\left(\left(\xi_{1}, \xi_{2}\right) \in E\right) \text {, }
$$

for some $\alpha \in R$. Since for any such $\Phi_{\alpha}$ we have $\left\|\Phi_{\alpha}\right\|=|\alpha|+|1-\alpha|$, it is clear that $\left\|\Phi_{\alpha}\right\|=1$ for all $\alpha$ with $0 \leq \alpha \leq 1$, but for each $\lambda>1$ there exist exactly one $\alpha_{1}>1$ and one $\alpha_{2}<0$ such that 
$\left\|\Phi_{\alpha_{1}}\right\|=\left\|\Phi_{\alpha_{2}}\right\|=\lambda \quad$ (namely, $\alpha_{1}=(\lambda+1) / 2$ and $\left.\alpha_{2}=(1-\lambda) / 2\right)$.

Let us observe that such an example is possible only for $\operatorname{codim} G=1$; more precisely, if $\operatorname{codim} G \geq 2$, then for each $\lambda>\|\varphi\|$ there exist an infinity of functionals $\Phi \in E^{*}$ satisfying (1). Indeed, in this case, for $G^{\perp}=\left\{\Psi \in E^{*}|\Psi|_{G}=\right.$ of we have

$$
\operatorname{dim} G^{\perp}=\operatorname{dim} E / G=\operatorname{codim} G \geq 2 ;
$$

but $\left\{\Phi \in E^{*}|\Phi|_{G}=\varphi\right\}=\Phi_{0}+G^{\perp}$, where $\Phi_{0} \in E^{*},\left.\Phi_{0}\right|_{G}=\varphi$, and hence, since $\Phi_{0}+G^{\perp}$ is a linear manifold with $\operatorname{dim}\left(\Phi_{0}+G^{\perp}\right) \geq 2$ and $\operatorname{dist}\left(0, \Phi_{0}+G^{\perp}\right)=\inf _{\Phi \in \Phi_{0}+G^{\perp}}\|\Phi\|=\|\varphi\|$, it follows that for each $\lambda>\|\varphi\|$ the set

$$
\left\{\Phi \in E^{*}|\Phi|_{G}=\varphi,\|\Phi\|=\lambda\right\}=\left(\Phi_{0}+G^{\perp}\right) \cap\left\{\Phi \in E^{*} \mid\|\Phi\|=\lambda\right\}
$$

is infinite, which proves our assertion.

Similar examples and remarks can be also given for the results below, but we shall not mention them again in the sequel.

COROLLARY 1. Let $E$ be a linear space, $\|\cdot\|$ con asymmetric norm on $E, G$ a linear subspace of $E, x_{0}$ an element of $E$ with

$\left(G \oplus\left[x_{0}\right]\right)^{\perp} \neq\{0\}$ and with $d=\inf _{g \in G}\left\|x_{0}-g\right\|>0$, and let $\lambda \geq 1 / d$. Then there exists $\Phi \in E^{*}$ such that

$$
\begin{aligned}
\Phi(g) & =0 \quad(g \in G), \\
\Phi\left(x_{0}\right) & =1, \\
\|\Phi\| & =\lambda .
\end{aligned}
$$

Moreover, if $\lambda>1 / d$, then there exist at least two distinct functionals $\Phi \in E^{*}$ satisfying (6)-(8).

Proof. Define a functional $\varphi$ on $G \oplus\left[x_{0}\right]=\left\{g+\alpha x_{0} \mid g \in G, \alpha \in R\right\}$ by 


$$
\varphi\left(g+\alpha x_{0}\right)=\alpha \quad(g \in G, \alpha \in R)
$$

Then $\varphi$ is linear on $G \oplus\left[x_{0}\right]$ and $\varphi(g)=0(g \in G), \varphi\left(x_{0}\right)=1$. Now let $g+\alpha x_{0} \in G \oplus\left[x_{0}\right],\left\|g+\alpha x_{0}\right\| \leq 1$. If $\alpha>0$, then

$$
I \geq\left\|g+\alpha x_{0}\right\|=\alpha\left\|(I / \alpha) g+x_{0}\right\| \geq \alpha d=\varphi\left(g+\alpha x_{0}\right) d ;
$$

on the other hand, if $\alpha \leq 0$, then

$$
I \geq\left\|g+a x_{0}\right\| \geq 0 \geq a d=\varphi\left(g+\alpha x_{0}\right) d \text {, }
$$

whence, since $g+\alpha x_{0} \in G \oplus\left[x_{0}\right]$ with $\left\|g+\alpha x_{0}\right\| \leq 1$ was arbitrary, we obtain $\|\varphi\| \leq 1 / d$ (actually, we shall see in Remark 1 (a) that $\|\varphi\|=1 / d$, but we do not need this here). Then, since $\left(G \oplus\left[x_{0}\right]\right)^{1} \neq\{0\}, \lambda \geq 1 / d \geq\|\varphi\|$, by Theorem 1 there exists $\Phi \in E^{*}$ satisfying $\left.\Phi\right|_{G \oplus\left[x_{0}\right]}=\varphi,\|\Phi\|=\lambda$, whence also $(6)-(8)$. Finally, if $\lambda>1 / d(\geq\|\varphi\|)$, then, again by Theorem 1 there exist at least two distinct functionals $\Phi \in E^{*}$ with these properties, which completes the proof of Corollary 1 .

REMARK 1. (a) The assumption that $G$ is not a hyperplane is necessary only for the case when $\lambda>1 / d$. Indeed, when $G$ is a hyperplane and $\lambda=1 / d$, the above proof yields $\varphi \in\left(G \oplus\left[x_{0}\right]\right)^{*}=E^{*}$ satisfying $\varphi(g)=0(g \in G), \varphi\left(x_{0}\right)=1$ and $\|\varphi\| \leq 1 / d$. But, taking $g_{n} \in G$ such that $\left\|x_{0}-g_{n}\right\| \rightarrow d$, we obtain

$$
1=\varphi\left(x_{0}-g_{n}\right) \leq\|\varphi\|\left\|x_{0}-g_{n}\right\| \rightarrow\|\varphi\| d,
$$

whence $\|\varphi\| \geq 1 / d$, and thus $\|\varphi\|=1 / d=\lambda$.

(b) From Remark 1 (a) it follows that if $B$ is a hyperplane in $E$, given by

$$
H=\left\{y \in E \mid \Phi_{0}(y)=c\right\},
$$

where $\Phi_{0} \in E^{*}, \Phi_{0} \neq 0, c \in R$ and if $x_{0} \in E$ is such that $\Phi_{0}\left(x_{0}\right) \geq c$, then the "asymmetric distance" from $x_{0}$ to $H$ is

$$
\operatorname{dist}_{\|\cdot\|}\left(x_{0}, H\right)=\inf _{y \in H}\left\|x_{0}-y\right\|=\left(\Phi_{0}\left(x_{0}\right)-c\right) /\left\|\Phi_{0}\right\| .
$$


Indeed, assume first that $c=0$. Then, by Remark 1 (a), there exists $\Phi \in E^{*}$ satisfying $\Phi(y)=0(y \in H), \Phi\left(x_{0}\right)=1$ and $\|\Phi\|=1 /\left(\right.$ dist $\left._{\|\cdot\|}\left(x_{0}, H\right)\right)$. By the first one of these relations and (10) with $c=0$, it follows that $\Phi_{0}=\alpha \Phi$ for some $\alpha \in R$. But then, $0=c \leq \Phi_{0}\left(x_{0}\right)=\alpha \Phi\left(x_{0}\right)=\alpha$, whence $\left\|\Phi_{0}\right\|=\Phi_{0}\left(x_{0}\right)\|\Phi\|$ and

$$
\operatorname{dist}_{\|\cdot\|}\left(x_{0}, H\right)=1 /\|\Phi\|=\Phi_{0}\left(x_{0}\right) /\left\|\Phi_{0}\right\| \text { ? }
$$

which proves (11) for $c=0$. Now, if $c \in R$ is arbitrary, then, from the result for $c=0$, proved above, we obtain, taking any $y_{0} \in H$ (thus, $H-y_{0}=\left\{y \in E \mid \Phi_{0}(y)=0\right\}$ and $\left.\Phi_{0}\left(x_{0}-y_{0}\right)=\Phi_{0}\left(x_{0}\right)-c \geq 0\right\}$, that $\operatorname{dist}_{\|\cdot\|}\left(x_{0}, H\right)=\operatorname{dist}_{\|\cdot\|}\left(x_{0}-y_{0}, H-y_{0}\right)=\Phi_{0}\left(x_{0}-y_{0}\right) /\left\|\Phi_{0}\right\|=\left(\Phi_{0}\left(x_{0}\right)-c\right) /\left\|\Phi_{0}\right\|$, which completes the proof of (11). Let us observe that (11) can be also proved directly, similarly to the particular case of (symmetric) norms (see, for example, [7], Chapter I, Lemma 1.2). Note also that if $\Phi_{0}\left(x_{0}\right) \leq c$, then

$$
\operatorname{aist}_{\|\cdot\|}\left(x_{0}, H\right)=\left(c-\Phi_{0}\left(x_{0}\right)\right) /\left\|-\Phi_{0}\right\| \text {. }
$$

Indeed, by (10), we have $H=\left\{y \in E \mid\left(-\Phi_{0}\right)(y)=-c\right\}$ and, by $\Phi_{0}\left(x_{0}\right) \leq c$ we have $\left(-\Phi_{0}\right)\left(x_{0}\right) \geq-c$, so we can apply (11) to $-\Phi_{0}$ and $-c$ and thus obtain (12). As was already mentioned, in general $\left\|-\Phi_{0}\right\| \neq\left\|\Phi_{0}\right\|$.

REMARK 2. By Remark 1 (b), we can give the following geometric interpretation of Corollary 1: if $E$ is a linear space, $\|\cdot\|$ an asymmetric norm on $E, G$ a linear subspace of $E$, and if $x_{0}$ is in element of $E$, with $\left(G \oplus\left[x_{0}\right]\right)^{\perp} \neq\{0\}$ and with $d=\operatorname{dist}_{\|\cdot\|}\left(x_{0}, G\right)>0$, then for every $\lambda \in R$ with $0<1 / \lambda \leq d$ there exists a hyperplane $H$ containing $G$ and such that dist $\|\cdot\|\left(x_{0}, H\right)=1 / \lambda$ (or, equivalently, containing $G$ and supporting the "asymmetric ball" $\left.B\left(x_{0}, 1 / \lambda\right)=\left\{y \in E \mid\left\|x_{0}-y\right\| \leq 1 / \lambda\right\}\right) ;$ moreover, if $0<1 / \lambda<d$, then there exist at least two such hyperplones $H$. Indeed, if $\Phi \in E^{*}$ is as in Corollary 1, then the hyperplane 


$$
H=\{y \in E \mid \Phi(y)=0\}
$$

contains $G$ and, by Kemark 1 (b), dist $\|\cdot\|\left(x_{0}, H\right)=\Phi\left(x_{0}\right) /\|\Phi\|=1 / \lambda$. Conversely, every hyperplane $H$ containing $G$, but not $x_{0}$ (if $x_{0} \in H$, then dist $\left._{\|\cdot\|}\left(x_{0}, H\right)=0\right)$, is of the form (13) for some $\Phi \in E^{*}$ with $\Phi\left(x_{0}\right) \neq 0$, so we may assume (considering $\left(1 / \Phi\left(x_{0}\right)\right) \Phi$ instead of $\Phi$, if necessary) that $\Phi\left(x_{0}\right)=1$. Thus, if $\operatorname{dist}_{\|\cdot\|}\left(x_{0}, H\right)=1 / \lambda$, then, by Remark I (b), $1 /\|\Phi\|=\Phi\left(x_{0}\right) /\|\Phi\|=$ dist $_{\|\cdot\|}\left(x_{0}, H\right)=1 / \lambda$, whence $\|\Phi\|=\lambda$, so $\Phi$ satisfies $(6)-(8)$. This result may be regarded as a complement to a theorem of Eidelheit [3], which states (for symmetric norms, but the result remains valid for asymmetric norms, too) that

$$
\operatorname{dist}_{\|\cdot\|}\left(x_{0}, G\right)=\sup _{\substack{H \in H \\ H \supset G}} \operatorname{dist}_{\|\cdot\|}\left(x_{0}, H\right) \text {, }
$$

where $H$ denotes the collection of all hyperplanes in $E$.

\section{Separation with double support}

THEOREM 2. Let $(E,|\cdot|)$ be a normed linear space, $U$ a bounded convex subset of $E$ with Int $U \neq \varnothing$, and $V$ a linear manifold in $E$ for which $\bar{V}$ is neither $E$, nor a hyperplane, such that

$$
V \cap \text { Int } U=\emptyset \text {. }
$$

Then there exists $\Phi \in E^{*}$ with $\Phi \neq 0$, such that

$$
\Phi(y)=\sup \Phi(U) \quad(y \in V) \text {. }
$$

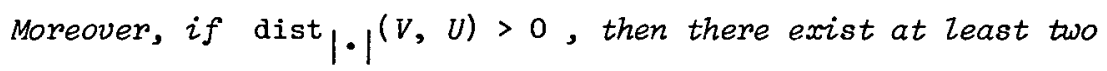
distinct functionals $\Phi \in E^{*}$ with $\Phi \neq 0$, satisfying (16).

Proof. We may assume that $0 \in$ Int $U$. Indeed, if the theorem is true in this case, then, since for any $z \in \operatorname{Int} U$ we have $0 \in \operatorname{Int}\left(U_{-z}\right)$ and $(V-z) \cap \operatorname{Int}(U-z)=\emptyset$ (respectively, dist $|\cdot|(V-z, U-z)>0)$, we obtain $\Phi \in E^{*}$ (respectively, at least two $\Phi \in E^{*}$ ), with $\Phi \neq 0$, such that $\Phi(y-z)=\sup \Phi(U-z) \quad(y \in V)$, whence $\Phi$ satisfies also (16).

Thus, assume that $0 \in$ Int $U$. Then the Minkowski functional 


$$
\|x\|=\inf _{\substack{\mu>0 \\ x \in \mu U}} \mu(x \in E),
$$

of $U$, is an asymmetric norm on $E$, and, since

$$
\text { Int } U=\{x \in E \mid\|x\|<1\} \neq \emptyset
$$

and $U$ is bounded, $\|\cdot\|$ is equivalent to the initial norm $|\cdot|$ on $E$. Furthermore, since $V$ is a linear manifold, we can write $V=x_{0}+G$, where $x_{0} \in V$ and $G$ is a linear subspace of $E$. Then, since $\bar{V}$ is neither $E$, nor a hyperplane, we have $\left(G \oplus\left[x_{0}\right]\right)^{\perp} \neq\{0\}$. Also, by (15), we have $d=\inf _{g \in G}\left\|x_{0}-g\right\|=\inf _{y \in V}\|y\| \geq 1$. Hence, by Corollary 1 above (with $\lambda=1 \geq 1 / d)$, there exists $\Phi \in E^{*}$ satisfying (6), (7) and $\|\Phi\|=1$. Then, by (7) and (6), we have $\Phi(y)=1\left(y \in x_{0}+G=V\right)$. On the other hand, $\sup \Phi(U)=\sup \Phi(x)=\|\Phi\|=1$ and hence $(16)$ holds. $\underset{\|x\| E}{x \in 1}$

Finally, if dist $|\cdot|(V, U)>0$, then, since $\|\cdot\|$ and $|\cdot|$ are equivalent, we have $\operatorname{dist}_{\|\cdot\|}(V,\{x \in E \mid\|x\|<I\})>0$, whence $d=\inf _{g \in G}\left\|x_{0}-g\right\|=\inf _{y \in V}\|y\|>1$. Consequently, by Corollary 1 (with $\lambda=1>1 / d)$, there exist at least two distinct functionals $\Phi \in E^{*}$ satisfying (6), (7) and $\|\Phi\|=1$. But then, as above, both of these $\Phi$ satisfy (16), which completes the proof of Theorem 2.

REMARK 3. (a) Theorem 2 admits the following geometric interpretation: if $U$ is a bounded convex subset, with Int $U \neq \emptyset$, of a normed linear space $(E,|\cdot|)$ and $V$ a linear manifold in $E$, with $\bar{V}$ neither $E$, nor a hyperplane, such that (15) holds, then there exists a hyperplane $H$ supporting $U$ and containing $V ;$ moreover, if dist $|\cdot|(V, U)>0$ then there exist at least two distinct hyperplanes $H$ supporting $U$ and containing $V$. Indeed, if $\Phi \in E^{*}$ is as in Theorem 2, then the hyperplane

$$
H=\{y \in E \mid \Phi(y)=\sup \Phi(U)\}
$$

supports $U$ and contains $V$. Conversely, every hyperplane $H$ which supports $U$ is of the form (18) for some $\Phi \in E^{*}, \Phi \neq 0$, so if $V \subset H$, 
then we must have (16).

(b) Theorem 2 is a complement to Mazur's separation theorem, which asserts (see, for example, [5], Chapter III, \$17) that if $U$ is a convex subset (not necessarily bounded) of $E$, with Int $U \neq \varnothing$, and if $V$ is a linear manifold in $E$, with $\bar{V} \neq E$, for which (15) holds, then there exist $\Phi \in E^{*}, \Phi \neq 0$, and $c \in R$, such that $\Phi(y)=c \geq \sup \Phi(U)$ $(y \in V)$, or, equivalently, there exists a hyperplane $H$ containing $V$ and such that $H \cap$ Int $U=\varnothing$.

In the particular case when $V$ is a single point, from Theorem 2 we obtain

COROLLARY 2. Let $E$ be a normed linear space, $U$ a bounded convex subset of $E$ with Int $U \neq \varnothing$, and $x \in E \backslash($ Int $U)$. Then there exists $\Phi \in E^{*}$ with $\Phi \neq 0$, such that

$$
\Phi(x)=\sup \Phi(U) .
$$

Moreover, if $x \in E \backslash \bar{U}$, then there exist at least two distinct functionals $\Phi \in E^{*}$ with $\Phi \neq 0$, satisfying (19).

REMARK 4. (a) Corollary 2 admits the following geometric interpretation: if $U$ is a bounded convex subset, with Int $U \neq \emptyset$, of $a$ normed linear space $E$ and if $x \in E \backslash$ (Int $U$ ), then there exists a hyperplane $H$ supporting $U$ and containing $x$; moreover, if $x \in E \backslash \bar{U}$, then there exist at least two distinct hyperplanes $H$ supporting $U$ and containing $x$.

(b) Corollary 2 is a complement to Mazur's support theorem, which asserts (see, for example, [1], Chapter I, $\$ 6$, Theorem 3) that if $U$ is a convex subset (not necessarily bounded) of $E$, with Int $U \neq \emptyset$, and if $x \in E \backslash($ Int $U)$, then there exists $\Phi \in E^{*}$ with $\Phi \neq 0$, such that $\Phi(x) \geq \sup \Phi(U)$, or, equivalently, there exists a hyperplane $H$ containing $x$ and such that $H \cap$ Int $U \neq \emptyset$.

THEOREM 3. Let $(E,|\cdot|)$ be a normed linear space, $A$ a bounded convex subset of $E$ with Int $A \neq \emptyset$, and $B$ a bounded convex subset of $E$, such that $B \neq \varnothing$ and

$$
B \cap \text { Int } A=\emptyset \text {. }
$$

Then there exists $\Phi \in E^{*}$ with $\Phi \neq 0$, such that 
(21)

$$
\sup \Phi(A)=\inf \Phi(B) \text {. }
$$

Moreover, if $\operatorname{dist}|\cdot|(B, A)>0$, then there exist at least two distinct functionals $\Phi \in E^{*}$ with $\Phi \neq 0$, satisfying (21).

Proof. Let

$$
U=(\operatorname{Int} A)-B=\{y-z \mid y \in \operatorname{Int} A, z \in B\}, x=0 .
$$

Then, clearly, $U$ is a bounded convex subset of $E$ and $U$ is open (since $U=\underset{z \in B}{U}\{$ Int $A-z\}$ ), so $U=\operatorname{Int} U \neq \emptyset$. Also, by (20), we have $x=0 \in E \backslash U$. Hence, by Corollary 2, there exists $\Phi \in E^{*}$ with $\Phi \neq 0$, such that $0=\Phi(0)=\sup \Phi\{($ Int $A)-B\}$, so in order to prove (2l), it will be enough to show that

$$
\sup \Phi\{(\operatorname{Int} A)-B\}=\sup \Phi(\operatorname{Int} A)-\inf \Phi(B)
$$

(since $\sup \Phi(A)=\sup \Phi(\operatorname{Int} A))$. Now, since $\Phi(y) \leq \sup \Phi(\operatorname{Int} A)$ $(y \in \operatorname{Int} A), \Phi(z) \geq \inf \Phi(B) \quad(z \in B)$, we have the inequalty $\leq$ in (23). On the other hand, for each $\varepsilon>0$ there exist $y_{\varepsilon} \in$ Int $A$ and $z_{\varepsilon} \in B$ such that $\Phi\left(y_{\varepsilon}\right) \geq \sup \Phi(A)-\varepsilon, \Phi\left(z_{\varepsilon}\right) \leq$ inf $\Phi(B)+\varepsilon$, whence, since $\varepsilon>0$ was arbitrary, we obtain the inequality $\geq$ in (23). Thus

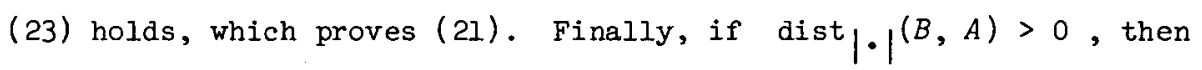
$\operatorname{dist}|\cdot|(B$, Int $A)>0$, whence $0 \$ \overline{(\operatorname{Int} A)-B}$. Thus, by Corollary 2, there exist at least two distinct functionals $\Phi \in E^{*}$ with $\Phi \neq 0$, satisfying $0=\Phi(0)=\sup \Phi\{(\operatorname{Int} A)-B\}$, whence also (21), which completes the proof of Theorem 3 .

REMARK 5. (a) Theorem 3 admits the following geometric interpretation: if $A, B$ are bounded convex subsets, with Int $A \neq \emptyset$, $B \neq \emptyset$, of a normed linear space $(E,|\cdot|)$ and if (20) holds, then there exists a hyperplane $H$ separating $A$ and $B$ and supporting each of these sets; moreover, if dist $|\cdot|(B, A)>0$, then there exist at least two distinct hyperplanes $H$ with these properties. Indeed, if $\Phi \in E^{*}$ is as in Theorem 3, then the hyperplane $H$ defined by

$$
H=\{y \in E \mid \Phi(y)=\sup \Phi(A)\}
$$

separates $A$ and $B$ and supports $A$ and $B$. Conversely, every hyperplane $H$ which supports $A$ is of the form (24) for some $\Phi \in E^{*}$, 
$\Phi \neq 0$. If $H$ supports also $B$, then we must have either $\sup \Phi(A)=\sup \Phi(B)$, or $\sup \Phi(A)=$ inf $\Phi(B)$; but, if $H$ also separates $A$ and $B$, then we can have only the second equality, so (2l) holds.

(b) Theorem 3 is a complement to Eidelheit's separation theorem, which asserts (see, for example, [1], Chapter I, $\$ 6$, Theorem 4) that if $A, B$ are convex subsets (not necessarily bounded) of $E$, with Int $A \neq \emptyset$, $B \neq \varnothing$, and such that (20) holds, then there exists $\Phi \in E^{*}$ with $\Phi \neq 0$ such that $\sup \Phi(A) \leq$ inf $\Phi(B)$, or, equivalently, there exists a hyperplane $H$ separating $A$ and $B$.

REMARK 6. (a) As shown by simple examples (for example, halfspaces), the assumptions of boundedness cannot be omitted in the results of $\S 3$.

(b) Naturally, one can also give direct proofs of the geometric results of $\S 3$ (without using $\S 2$ ). Moreover, as in the classical theory (see, for example, [5]) one can show that the results of $\S 2$ and $\S 3$ are equivalent.

\section{References}

[1] Mahlon M. Day, Normed Zinear spaces, 3rd ed. (Ergebnisse der Mathematik und ihrer Grenzgebiete, 21. Springer-Verlag, Berlin, Heidelberg, New York, 1973).

[2] R.J. Duffin and L.A. Karlovitz, "Formulation of linear programs in analysis. I: Approximation theory", SIAM J. AppZ. Math. 16 (1968), 662-675.

[3] M. Eidelheit, "Quelques remarques sur les fonctionnelles linéaires", Studia Math. 10 (1948), 140-147.

[4] Victor Klee, "Separation and support properties of convex sets - a survey", Control theory and the calculus of variations, 235-303 (Academic Press, New York and London, 1969).

[5] Gottfried Köthe, Topologische Zineare Räume. I (Die Grundlehren der mathematischen Wissenschaften, 107. Springer-Verlag, Berlin, Göttingen, Heidelberg, 1960). 
[6] М.Г. Нрейн, "L-проблема в абстрантном линейном нормированном пространстве", О некоторих вопросах теории моментов, 171-199 (Научно- Твхнмчесное Кздательство Унранны, Харьнов, 1938). M. Kre In, "The $L$-problem in an abstract linear normed space", Some questions in the theory of moments, 175-204 (translated by W. Fleming and D. Prill. Translations of Mathematical Monographs, 2. American Mathematical Society, Providence, Rhode Island, 1962).

[7] Ivan Singer, Best approximation in normed linear spaces by elements of Zinear subspaces (Die Grundlehren der mathematischen Wissenschaften, 171. Publishing House of the Academy of the Socialist Republic of Romania, Bucharest; Springer-Verlag, Berlin, Heidelberg, New York, 1970).

[8] Ivan Singer, "Minimization of continuous linear functionals on complements of convex subsets of locally convex spaces", Math. Operationsforsch. Statist. Ser. Optim. (to appear).

Institutul Nafional pentru Creafie Ştiinfifică şi Tehnică, București, Romania; Institutul de Matematica, Bucuresti, Romania. 